\title{
ESTUDO RETROSPECTIVO DE CRIANÇAS E JOVENS COM DEFICIÊNCIA AUDITIVA: CARACTERIZAÇÃO DAS ETIOLOGIAS E QUADRO AUDIOLÓGICO
}

\section{Retrospective study of hearing impaired children and teenager: characterizing the etiologies and audiological aspects}

\author{
Altair Cadrobbi Pupo (1), Clay Rienzo Balieiro ${ }^{(2)}$, Renata de Souza Lima Figueiredo ${ }^{(3)}$
}

\begin{abstract}
RESUMO
Objetivo: conhecer os fatores de risco para deficiência auditiva e obter informações sobre o tempo transcorrido entre suspeita, diagnóstico e intervenção em crianças e jovens deficientes auditivos atendidos no Serviço de Audiologia Educacional, DERDIC-PUC-SP. Métodos: estudo retrospectivo de 162 prontuários de deficientes auditivos com idade entre um e 17 anos e 6 meses, atendidos de 1999 a 2002. Para registro dos dados utilizou-se a adaptação do protocolo elaborado no Fórum de Reabilitação Aural do Encontro Internacional de Audiologia, no Brasil. Resultados: $54 \%$ dos sujeitos eram do sexo masculino e $46 \%$ do feminino; $60 \%$ tinham idade entre três e oito anos e 11 meses; $43 \%$ tiveram a suspeita da deficiência auditiva no primeiro ano de vida. Em $25 \%$ dos casos, o diagnóstico ocorreu logo após a suspeita, em 34\% ocorreu um intervalo de até um ano; $11 \%$ iniciaram atendimento fonoaudiológico após diagnóstico, 54\% demoraram até um ano e $27 \%$ demoraram mais de um ano. Trinta e dois por cento apresentaram etiologia desconhecida, $18 \%$ genética, $17 \%$ presumida multifatorial, 15\% meningite, $9 \%$ rubéola congênita. Conclusão: a prevalência da etiologia desconhecida aponta para a necessidade do aprofundamento no diagnóstico etiopatológico (estudos genéticos, de imagens e laboratoriais) como rotina para se obter as causas da deficiência auditiva. Observou-se que mesmo após as campanhas de vacinação, a rubéola ainda foi a maior causa de deficiência auditiva congênita e a meningite a maior causa das deficiências auditivas adquiridas após o nascimento. Constatou-se um longo intervalo de tempo entre suspeita, confirmação e início da intervenção fonoaudiológica.
\end{abstract}

DESCRITORES: Diagnóstico; Surdez / etiologia; Fatores de Risco; Perda Auditiva

\section{INTRODUÇÃO}

Estudiosos da área da saúde têm buscado conhecer indicadores epidemiológicos de forma a

(1) Fonoaudióloga da Clínica Eco; Professora Associada da Faculdade de Fonoaudiologia da Pontifícia Universidade Católica de São Paulo; Coordenadora do Curso de Especialização em Fonoaudiologia - Audição da Pontifícia Universidade Católica de São Paulo; Coordenadora Geral de Estágio da Pontifícia Universidade Católica de São Paulo; Doutora em Distúrbios da Comunicação Humana pela Universidade Federal de São Paulo.

(2) Fonoaudióloga da Clínica Eco; Professora Associada da Faculdade de Fonoaudiologia da Pontifícia Universidade Católica de São Paulo; Fonoaudióloga do Serviço Audiologia Educacional - Divisão de Educação e Reabilitação dos Distúrbios da Comunicação da Pontifícia Universidade Católica de São Paulo; Doutora em Distúrbios da Comunicação Humana pela Universidade Federal de São Paulo.

(3) Fonoaudióloga; Mestre em Fonoaudiologia pela Pontifícia Universidade Católica de São Paulo. exercer ações promotoras da saúde para a população em geral. A população infantil tem merecido especial atenção no campo da saúde auditiva. Subsidiar a proposição de ações de promoção da saúde auditiva na infância quer sejam preventivas ou para tratamento de problemas auditivos é meta dos otorrinolaringologistas, pediatras e fonoaudiólogos. Conhecer os indicadores referentes a problemas de audição na infância contribui para o desenvolvimento das ações de promoção de saúde e prevenção de agravos, além de possibilitar a realização de diagnósticos mais precoces.

A importância dos primeiros anos de vida para o desenvolvimento da criança e especialmente da linguagem exige que o diagnóstico da deficiência de audição seja realizado o mais cedo possível, preferencialmente antes dos seis meses de vida. Conhecer as principais causas da deficiência auditiva contribui tanto para a prevenção quanto para a 
detecção. Em estudo ${ }^{1}$ realizado, foi constado que a causa da deficiência auditiva foi desconhecida em $36 \%$ dos sujeitos estudados e presumida em $63 \%$, sendo que em $38 \%$ dos sujeitos o intervalo de tempo transcorrido entre a suspeita e a confirmação da deficiência auditiva foi de mais de dois anos. Em outro estudo ${ }^{2}$ realizado na cidade de Bauru/ São Paulo, observou-se que a suspeita da perda auditiva antes dos seis meses ocorreu em $22,5 \%$ dos sujeitos. A idade do primeiro diagnóstico variou do nascimento a 69 meses, com idade média de 20,17 meses e mediana de 18,72 meses. Somente em $16,48 \%$ dos sujeitos, a etiologia não pôde ser determinada. A etiologia genética foi observada em $20,65 \%$ dos casos e as etiologias ambientais, em $58,70 \%$. Os diversos fatores etiológicos, o diagnóstico tardio e o reconhecimento dos aspectos positivos da intervenção fonoaudiológica iniciada o mais cedo possível levaram os profissionais a buscar recursos para antecipar esse diagnóstico, já que a deficiência auditiva pode acometer o ser humano em qualquer idade.

O presente estudo, realizado em 2002, investigou aspectos relativos à população de crianças $e$ jovens deficientes auditivos em atendimento fonoaudiológico no Serviço de Audiologia Educacional, DERDIC-PUCSP, instituição da cidade de São Paulo - Brasil, seis meses antes da aprovação da Portaria da Saúde Auditiva. Nesse sentido, esse trabalho objetivou conhecer os fatores de risco, determinantes da alteração auditiva e obter informações sobre o tempo transcorrido entre a suspeita, diagnóstico e intervenção fonoaudiológica.

\section{MÉTODOS}

Caracterizou-se como um estudo retrospectivo com base em prontuários de 162 crianças e jovens com deficiência de audição, atendidos no Serviço de Audiologia Educacional, DERDIC/PUC-SP, no período de 1999 a 2002.

Para registro dos dados utilizou-se uma adaptação de protocolo elaborado no Fórum de Reabilitação Aural, que ocorre no Encontro Internacional de Audiologia, anualmente no Brasil.

A análise da relação entre a idade da confirmação (diagnóstico) da deficiência auditiva e a idade do início da intervenção fonoaudiológica foi realizada a partir de 67 protocolos, uma vez que nos demais essas informações não se encontravam disponíveis.

Os critérios utilizados para análise foram:

1) quanto à idade: manteve-se uma mesma data de preenchimento para todos os protocolos;

2) quanto à etiologia considerou-se: desconhecida - quando não existia hipótese diagnóstica sobre a causa da deficiência auditiva; presumida - quando existia hipótese diagnóstica, porém sem a confirmação através de exames específicos e/ou confirmação médica descrita em relatório; confirmada - quando as hipóteses diagnósticas estavam confirmadas por meio de exames específicos e/ou descritas em relatórios médicos;

3) quanto ao grau, obedeceu-se os critérios de Silman \& Silvermam ${ }^{3}-$ média dos limiares tonais nas freqüências de 0,5, 1 e $2 \mathrm{kHz}$ - a partir do exame audiológico mais recente;

4) quanto à simetria/assimetria da perda auditiva, seguiram-se os critérios de Newton \& Rowson ${ }^{4}$ - média das diferenças dos limiares auditivos entre as orelhas nas freqüências analisadas, média menor ou igual a $10 \mathrm{~dB}$, perda auditiva simétrica, média maior do que $10 \mathrm{~dB}$, assimétrica;

5) quanto ao padrão de variação, utilizaram-se os critérios de Pupo ${ }^{5}$ - estável, variação nos limiares tonais não ultrapassam $10 \mathrm{~dB}$; flutuante, piora e melhora do limiar tonal de pelo menos $15 \mathrm{~dB}$ entre cada audiometria, no mínimo em duas freqüências, sem, no entanto haver diferenças no limiar tonal, de pelo menos $15 \mathrm{~dB}$, entre a primeira e a última audiometria (o que caracterizaria a perda progressiva); progressiva, diferença para pior no limiar tonal de pelo menos $15 \mathrm{~dB}$ entre a primeira e a última audiometria, no mínimo em duas freqüências; flutuante/ progressiva, piora e melhora do limiar tonal de pelo menos $15 \mathrm{~dB}$ entre as audiometrias, com uma diferença no limiar tonal para pior de pelo menos 15 $\mathrm{dB}$ entre a primeira e a última audiometria, em pelo menos duas freqüências.

Este trabalho foi aprovado pelo Comitê de Ética do Programa de Estudos Pós Graduados em Fonoaudiologia, da PUC-SP sob o protocolo no. 001/2007 e, segundo a resolução no.196/96 (CNS).

\section{RESULTADOS}

De acordo com o levantamento dos dados dos 162 prontuários, a população em estudo caracterizou-se por $54 \%$ (88) de sujeitos do sexo masculino e $46 \%$ (74) do feminino. A idade variou entre 12 meses - 17 anos e seis meses com concentração de $60 \%$ (96) entre três e oito anos e 11 meses.

$\mathrm{Na}$ Figura 1, pode-se observar que houve suspeita da deficiência auditiva até o primeiro ano de vida em $43 \%$ (74) dos casos. A suspeita da deficiência de audição ocorreu, em 78\% (127) dos casos, pela família ou responsável, em 14\% (22) por profissionais da saúde, $2 \%$ (4) por profissionais da educação, $3 \%$ (4) por outras categorias e em 3\% (5) dos casos não foi possível obter essa informação. 


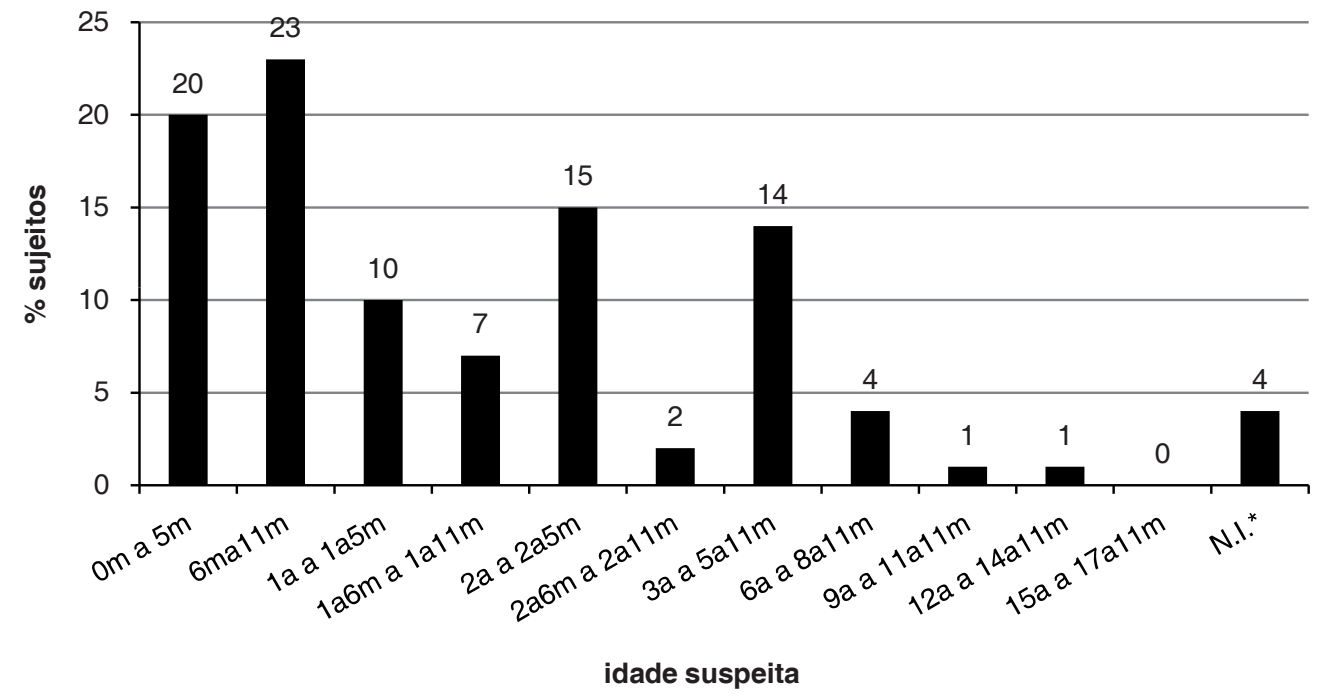

Figura 1 - Distribuição dos sujeitos segundo a idade da suspeita da deficiência auditiva ( $\mathrm{N}=162)$

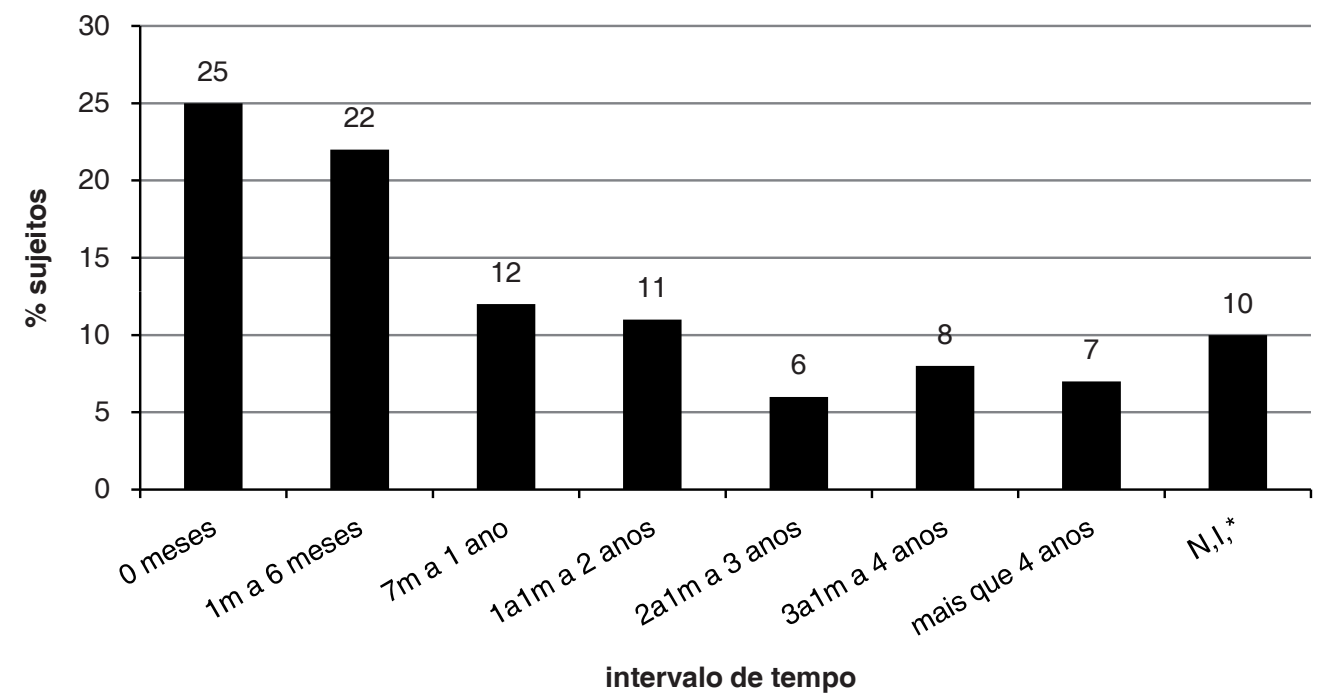

Figura 2 - Distribuição dos sujeitos segundo o intervalo de tempo entre a idade da suspeita e o diagnóstico $(\mathrm{N}=162)$

$\mathrm{Na}$ Figura 2, observa-se que em 25\% (40) dos casos estudados, o diagnóstico foi realizado imediatamente após a suspeita, enquanto em $34 \%$ (54) o diagnóstico ocorreu com intervalo de até um ano após a suspeita e em $25 \%$ (41) entre mais de um ano até 4 anos após a suspeita.

A Figura 3 mostra o intervalo de tempo entre o diagnóstico da deficiência auditiva e o início da intervenção fonoaudiológica. Onze por cento (8) iniciaram o atendimento logo após o diagnóstico, $54 \%$ (36) até um ano após e $27 \%$ (18) com intervalo maior que 1 ano.

A Figura 4 mostra que não houve diferença significativa quanto à época da suspeita no grupo de faixa etária até um ano e cinco meses, entre os casos com fatores de risco e aqueles sem fatores de risco para deficiência de audição. Essa diferença ocorreu no grupo da faixa etária de um ano e seis meses a dois anos de idade.

Quanto à etiologia, 32\% (52) apresentaram etiologia desconhecida, $41 \%$ (66) presumida e $27 \%$ (44) confirmada. Dentre as causas confirmadas e presumidas (Figura 5), encontrou-se 18\% (29) causa genética, $17 \%$ (27) presumida multifatorial, 15\% (24) meningite, 9\% (14) rubéola congênita, 4\% (6) medicações ototóxicas, $2 \%$ (4) hiperbilirrubinemia, $1 \%$ (2) baixo peso, 1\% (2) citomegalovirus e $1 \%(2)$ toxoplasmose.

Quanto ao grau (referente à melhor orelha), 68\% (110) apresentaram perda auditiva de grau severo e profundo (Figura 6). 


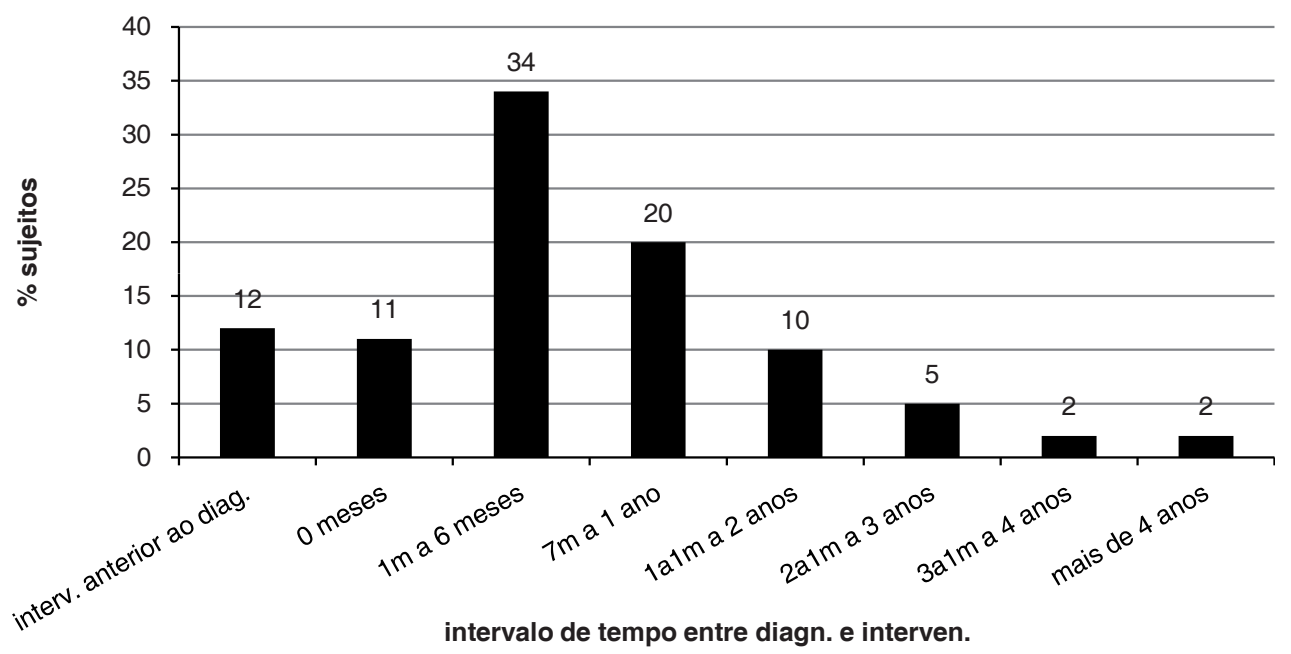

Figura 3 - Distribuição dos sujeitos segundo intervalo de tempo entre diagnóstico e início do atendimento fonoaudiologico $(\mathrm{N}=67)$

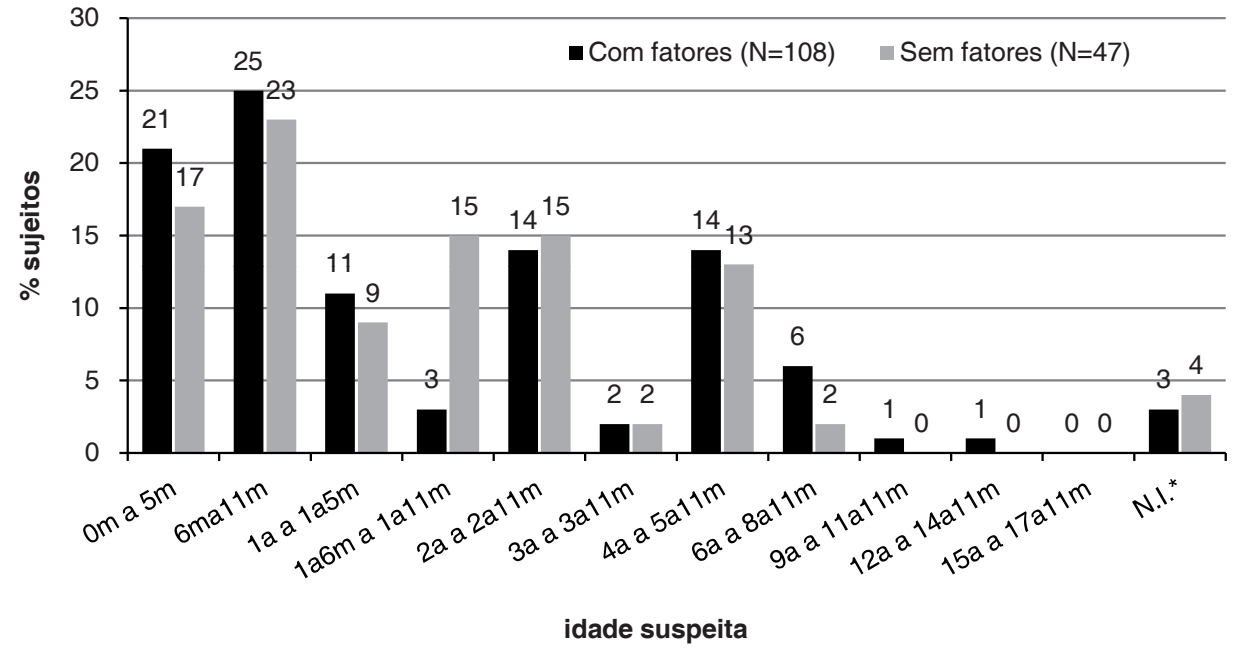

Figura 4 - Distribuição dos sujeitos segundo idade da suspeita da deficiência de audição e a presença ou não de fatores de risco para deficiência auditiva $(\mathrm{N}=162)$

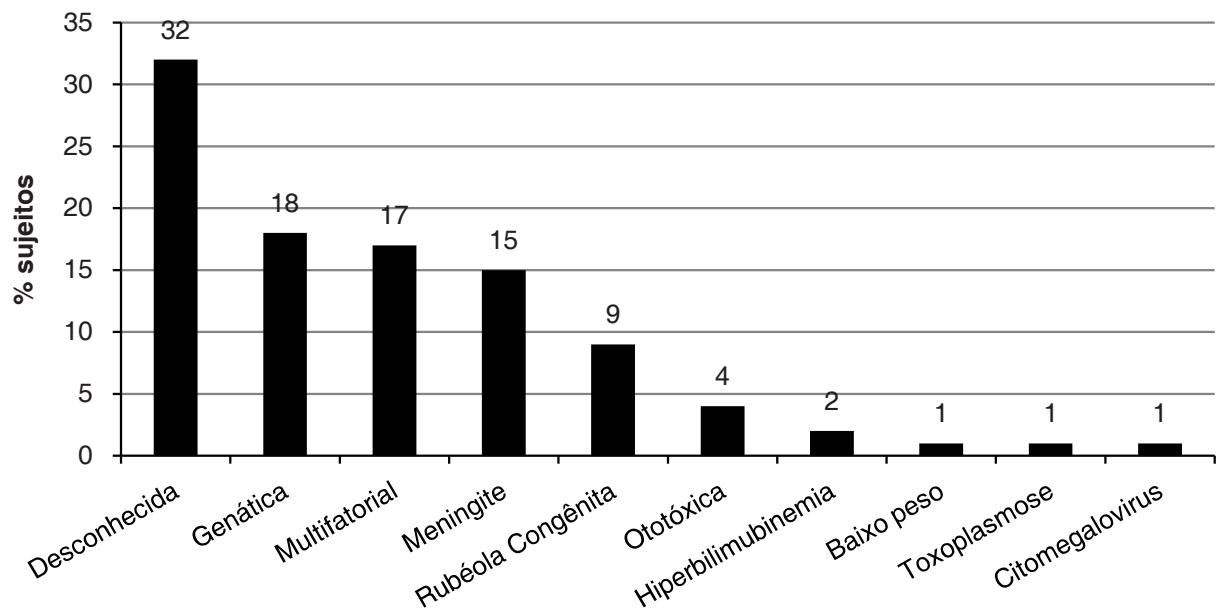

Figura 5 - Distribuição de sujeitos segundo a prevalência das etiologias da deficiência auditiva $(\mathrm{N}=162)$ 


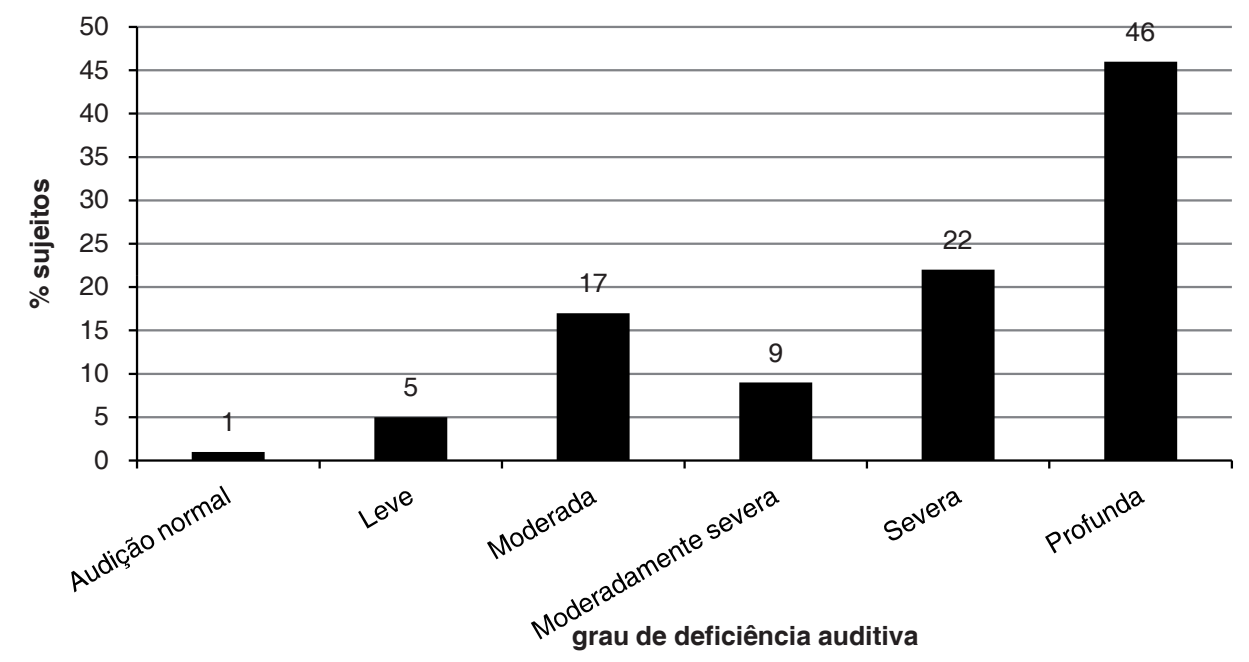

Figura 6 - Distribuição de sujeitos segundo o grau da perda auditiva da melhor orelha ( $\mathrm{N}=162)$

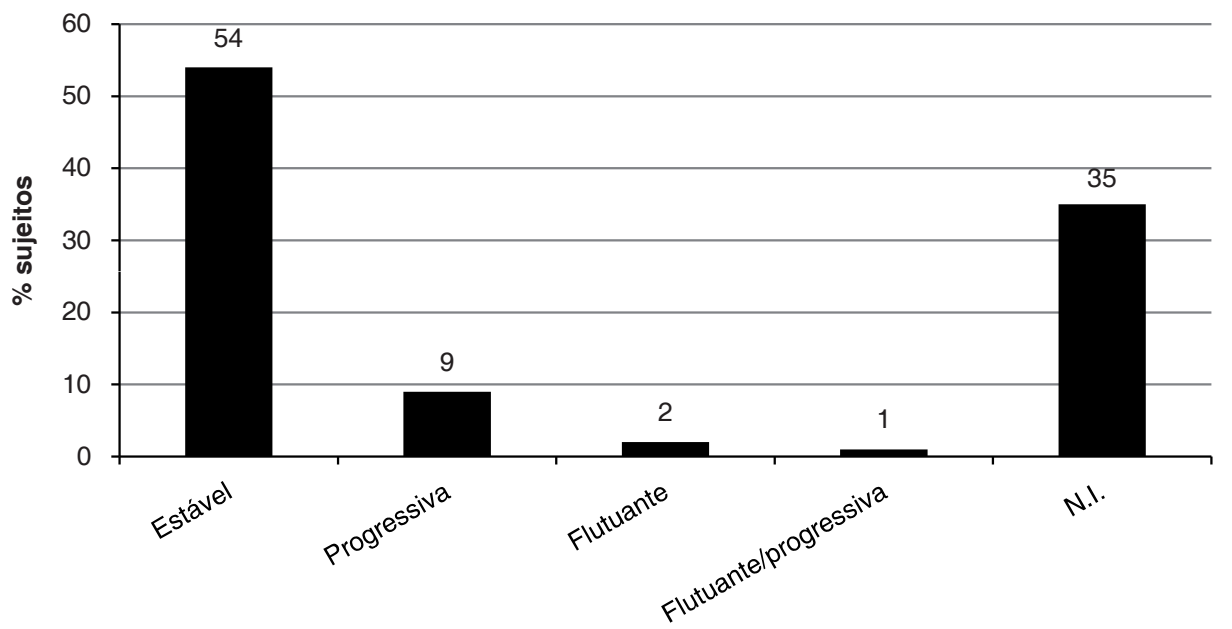

Figura 7 - Distribuição dos sujeitos segundo o padrão de variação da perda auditiva (N=162)

Em relação ao padrão de variação da perda auditiva (Figura 7), 12\% (18) das crianças apresentaram variação nos limiares tonais, sendo $9 \%$ (14) progressivas, $2 \%$ (3) flutuantes, e $1 \%$ (1) flutuante-progressiva.

Quanto ao tipo de perda auditiva, na orelha direita, 93\% (151) apresentaram perda auditiva sensorioneural, $1 \%$ (1) condutiva, $2 \%$ (4) mista, $2 \%$ (3) central, audição normal em uma orelha $1 \%$ (1) e $1 \%$ (2) ainda por esclarecer na época do estudo; na orelha esquerda $94 \%$ (152) apresentaram perda auditiva sensorioneural, 3\% (5) mista, 2\% (3) central e a esclarecer, na época do estudo, em 1\% (2).

\section{DISCUSSÃO}

Neste estudo encontrou-se 88 sujeitos (54\%) do sexo masculino e 75 (46\%) do sexo feminino o que está de acordo com dados da literatura nacional e internacional ${ }^{1,6,7}$ que mostram que a perda auditiva é mais freqüente no sexo masculino.

A idade da suspeita em $43 \%$ (70) dos sujeitos concentrou-se no primeiro ano de vida. Sessenta e sete por cento (108) apresentavam fator de risco para perda auditiva, entretanto, apenas em $46 \%$ suspeitou-se da deficiência de audição no primeiro ano de vida. Esses resultados sugerem que os fatores de risco presentes ao nascimento não foram valorizados como indicadores de possíveis alterações auditivas, o que justifica em parte, a implementação de programas de triagem auditiva neonatal universal, enquanto medida de atenção à saúde.

Em somente $25 \%$ dos casos estudados, o diagnóstico foi realizado imediatamente após a suspeita, enquanto em 34\% o diagnóstico ocorreu com intervalo de até um ano após a suspeita e em $25 \%$ entre um a quatro anos. Se, conforme observado, 


\begin{tabular}{|c|c|c|c|c|c|c|}
\hline \multirow[b]{2}{*}{ Figura 8} & \multicolumn{6}{|c|}{ Estudos Internacionais } \\
\hline & $\begin{array}{c}\text { Vartiainen et al, } \\
1997^{12} \\
\text { Finlândia }\end{array}$ & $\begin{array}{c}\text { Billings, Kenna } \\
1999^{19} \\
\text { EUA }\end{array}$ & $\begin{array}{l}\text { Uus,Davis, } \\
2000^{17} \\
\text { Estonia }\end{array}$ & $\begin{array}{l}\text { Walch et al, } \\
2000^{18} \\
\text { Austria }\end{array}$ & $\begin{array}{l}\text { Zakzouk, Al- } \\
\text { Anazy, } \\
2002^{13} \\
\text { Arábia Saudita }\end{array}$ & $\begin{array}{c}\text { Egeli et al, } \\
2003^{7} \\
\text { Istanbul }\end{array}$ \\
\hline Casos estudados & 98 & 301 & 248 & 106 & 302 & 162 \\
\hline Desconhecida & $30 \%$ & $31,9 \%$ & $34,3 \%$ & $44 \%$ & $12 \%$ & $20,98 \%$ \\
\hline $\begin{array}{l}\text { Hereditária } \\
\text { (genética) }\end{array}$ & $41 \%$ & $23 \%$ & $36,6 \%$ & $18 \%$ & $60 \%$ & $41,35 \%$ \\
\hline Rubéola & $3,06 \%$ & - & $1,2 \%$ & $3,77 \%$ & $3,22 \%$ & - \\
\hline citomegalovirus & $2,04 \%$ & - & $0,4 \%$ & - & - & - \\
\hline Toxoplasmose & - & - & $1,2 \%$ & - & - & - \\
\hline Meningite & $7,14 \%$ & $4,0 \%$ & $8,5 \%$ & $7,74 \%$ & $7,66 \%$ & $10,49 \%$ \\
\hline Ototóxicos & - & $2,3 \%$ & - & - & - & - \\
\hline Fatores Perinatais & $8,16 \%$ & $19,9 \%$ & $11,6 \%$ & $20 \%$ & $7,25 \%$ & $3,7 \%$ \\
\hline
\end{tabular}

Figura 8 - Resultados de pesquisas internacionais referentes à etiologia da deficiência auditiva

\begin{tabular}{|c|c|c|c|c|c|c|}
\hline \multirow[b]{2}{*}{ Figura 9} & \multicolumn{6}{|c|}{ Estudos Nacionais } \\
\hline & $\begin{array}{l}\text { Salerno et al, } \\
1985^{16} \\
\text { Brasília - DF }\end{array}$ & $\begin{array}{c}\text { Tabith et al, } \\
1989^{10} \\
\text { São Paulo,SP }\end{array}$ & $\begin{array}{l}\text { Walber et al, } \\
1995^{11} \\
\text { RS }\end{array}$ & $\begin{array}{c}\text { Nóbrega, } \\
2002^{1} \\
\text { São Paulo,SP }\end{array}$ & $\begin{array}{c}\text { Cabral dos } \\
\text { Reis, } 2006^{6} \\
\text { João } \\
\text { Pessoa,Pa }\end{array}$ & $\begin{array}{l}\text { Pupo, Balieiro, } \\
\text { Figueiredo } \\
2006 \\
\text { São Paulo,SP }\end{array}$ \\
\hline Casos estudados & 782 & 119 & 35 & 519 & 54 & 162 \\
\hline Desconhecida & $29,6 \%$ & $37,81 \%$ & $40 \%$ & $36,6 \%$ & $40,7 \%$ & $32 \%$ \\
\hline $\begin{array}{l}\text { Hereditária } \\
\text { (genética) }\end{array}$ & $53,73 \%$ & $16,8 \%$ & $5,71 \%$ & $18,2 \%$ & $9,3 \%$ & $18 \%$ \\
\hline Rubéola & $2,55 \%$ & $14,28 \%$ & $37,14 \%$ & $13,7 \%$ & $27,7 \%$ & $9 \%$ \\
\hline citomegalovirus & - & - & - & - & - & $1 \%$ \\
\hline Toxoplasmose & - & - & - & - & - & $1 \%$ \\
\hline Meningite & $4,73 \%$ & $10,08 \%$ & $8,57 \%$ & $10,6 \%$ & $13 \%$ & $15 \%$ \\
\hline Ototóxicos & $3,7 \%$ & $4,2 \%$ & $2,85 \%$ & - & $7,4 \%$ & $4 \%$ \\
\hline Fatores Perinatais & $2 \%$ & $10,92 \%$ & $2,85 \%$ & $11,7 \%$ & $1,9 \%$ & $3 \%$ \\
\hline Multifatoriais & - & $5,88 \%$ & - & - & $\ldots$ & $17 \%$ \\
\hline
\end{tabular}

Figura 9 - Resultados de pesquisas nacionais referentes à etiologia da deficiência auditiva

foi a família que suspeitou da deficiência auditiva em $78 \%$ dos casos estudados, pode-se considerar que muitos pais ficaram por um período significativo de tempo, aguardando uma resposta às suas suspeitas. A suspeita dos pais é um indicador importante aos profissionais da saúde.

O atendimento fonoaudiológico para $12 \%$ dessa população teve início antes da conclusão diagnóstica, para $11 \%$ ocorreu logo após o diagnóstico, para $54 \%$ até um ano após e para $27 \%$ com intervalo maior que um ano. Deve-se considerar que a amostra estudada refere-se a uma instituição de referência para diagnóstico e atendimento fonoaudiológico de crianças com deficiência auditiva na cidade de São Paulo, mas, apesar disso, $81 \%$ dos casos não iniciaram o atendimento imediatamente após o diagnóstico, indicando carência de programas específicos para atendimento clínico dessas crianças e suas famílias, o que também foi apon- tado em estudos nacionais ${ }^{2,6,8,9} \mathrm{em}$ diversas regiões do Brasil.

A etiologia desconhecida continua prevalecendo, corroborando com estudos ${ }^{1,10-13}$ conforme especificado nas Figuras 8 e 9.

Das causas genéticas, em $72 \%$ (21) a etiologia foi presumida e em $28 \%$ (8) foi confirmada, revelando $13 \%$ (1) de casos autossômicos recessivos, 13\% (1) herança mitocondrial, 38\% (3) síndromes (Goldenhar, Waadernburg e alargamento do aqueduto vestibular) e $38 \%$ (3) sem especificações quanto ao padrão de herança. Neste estudo, a meningite foi a maior responsável pela deficiência auditiva adquirida, assim como em outros trabalhos 1,11,12; enquanto a rubéola materna foi a maior causa da deficiência auditiva congênita adquirida, diferentemente de outros países em que após a introdução da vacina, o citomegalovirus passou a ser a principal causa. Por outro lado, encontrou-se, neste estudo, 
um caso de citomegalovirus confirmado e outro presumido, o que sugere necessidade de aprofundamento no diagnóstico etiológico. Considere-se ainda que em uma publicação ${ }^{10}$ mostra $11,6 \%$ de casos por rubéola e em outra ${ }^{1} 13,7 \%$. Infelizmente, a falta de padronização entre as pesquisas não permite que se avaliem os efeitos dos programas de vacinação durante esse período. O mapeamento genético, exames por imagem, exames laboratoriais podem esclarecer parte das deficiências auditivas que hoje são consideradas desconhecidas. $\mathrm{O}$ conhecimento sobre a prevalência das etiologias das deficiências auditivas auxilia na promoção de ações informativas e educativas à população e profissionais e na adoção de ações preventivas.

Quanto ao padrão de variação da perda auditiva, esse aspecto não foi avaliado em 35\% (57) dos casos devido ao número de avaliações audiológicas realizadas (não havendo possibilidade de comparálas). Cinqüenta e quatro por cento (87) apresentaram perda auditiva estável, $9 \%$ (14) progressão, 2\% (3) flutuação e 1\% (1) perda flutuante/progressiva. Atenção especial deve ser dada à ocorrência de perdas auditivas progressivas, principalmente por etiologia genética, o que demanda cuidados no processo terapêutico.

Prevaleceram as perdas auditivas do tipo sensorioneural $(93 \%)$, de grau profundo $(46 \%)$ e severo $(22 \%)$, simétricas $(72 \%)$, estáveis $(54 \%)$ e perda unilateral em apenas $1 \%$.

Os nove sujeitos com etiologia por rubéola congênita apresentaram perda auditiva do tipo sensorioneural ${ }^{14}, 7 \%$ (1) grau moderado, $36 \%$ (5) de grau severo e $57 \%$ (8) de grau profundo; $71 \%$ perda auditiva simétrica, $14 \%$ (2) perda progressiva e em $21 \%$ (3) não foi possível obter essa informação em função do número de audiometrias realizadas.

Nos casos de perda auditiva por meningite, $83 \%$ (20) adquiriram a perda auditiva no primeiro ano de vida, sendo $63 \%$ (15) de grau profundo. O tipo foi predominantemente sensorioneural, em ambas orelhas. Treze por cento (3) apresentaram perda progressiva bilateral e simétrica, achados que estão de acordo com outros estudos ${ }^{15}$.

\section{CONCLUSÃO}

De acordo com o material analisado pode-se afirmar que a etiologia desconhecida continua prevalecendo, o que mostra a necessidade de se realizar rotineiramente estudos genéticos de forma a se obter um perfil real da prevalência das causas das alterações auditivas congênitas; a meningite ainda é a responsável pela maior causa de deficiência auditiva adquirida; das etiologias congênitas adquiridas, a rubéola materna ainda foi a maior causa da deficiência auditiva; deve-se ressaltar que devido à faixa etária dessa população não foi possível avaliar, neste estudo, o impacto da vacinação contra rubéola; o intervalo de tempo entre a suspeita, a confirmação e o início de algum tipo de intervenção.

$\mathrm{Na}$ população estudada há uma prevalência de perdas auditivas bilaterais, simétricas de grau severo e profundo.

\section{ABSTRACT}

Purpose: to become aware of the risks factors for hearing impairment and describe the time gap between the suspicions of the hearing loss, the diagnostic and the beginning of the rehabilitation for a group of hearing impaired children and teenagers from an institution in São Paulo, Brazil - DERDIC PUC-SP, that took part in a rehabilitation program from 1999 to 2002. Methods: a retrospective study of 162 files of hearing impaired children between $12 \mathrm{mo}$ and 17:6 yrs old, the questionnaire elaborated by The Aural Rehabilitation Forum which occurs annually during the International Audiology Meeting in Brazil was used. Results: the results showed $54 \%$ male and $46 \%$ female subjects; $60 \%$ between 3 and $8: 11$ years old; $43 \%$ had the suspicions of the hearing loss in the first year of life; for $25 \%$ of the subjects the diagnostic was carried out just after the suspicions; $34 \%$ finished the diagnostic one year after the suspicions; $11 \%$ began rehabilitation just after the diagnostic, $54 \%$ after a year and $27 \%$ more than a year. $32 \%$ had unknown etiology, $18 \%$ genetic causes, $17 \%$ presumed multiple factors, $15 \%$ meningitis, $9 \%$ congenital rubella. Conclusion: the prevalence of unknown etiology shows the need for deepening the diagnosis, and as a routine, carry out genetics, images and laboratories studies, in order to know the etiologies of the hearing loss. Even though after implementing immunization campaigns, rubella was the most prevalent cause of the congenital hearing loss and meningitis was the most prevalent cause of acquired hearing loss. A precious time for the child development was lost because of the time gap between suspicions, diagnostic and rehabilitation.

KEYWORDS: Diagnosis; Deafness / etiology; Risk Factors; Hearing Loss 


\section{REFERÊNCIAS}

1. Nóbrega M. Deficiência auditiva na infância: a experiência do ambulatório de deficiência auditiva da UNIFESP/EPM. Anais do $17^{\circ}$ Encontro Internacional de Audiologia. Bauru; 2002.

2. Meyer ASA. Caracterização dos aspectos diagnósticos e de intervenção das crianças atendidas nos serviços de deficiência auditiva do HRAC/USPBauru. [doutorado]. Bauru (SP): Universidade de São Paulo; 2003.

3. Silman S, Silverman CA. Auditory diagnosis: principles and applications. San Diego: Singular Publishing Group; 1997. 428 p.

4. Newton VE, Rowson VJ. Progressive sensorineural hearing loss in childhood. Br J Audiol. 1988; 22(4):287-95.

5. Pupo CA. Deficiência auditiva progressiva em crianças e jovens: prevalência das hipóteses etiológicas e características audiológicas. Disturb Comun. 2002; 13:219-41.

6. Reis DC. Saúde auditiva em João Pessoa. [mestrado]. São Paulo (SP): Pontifícia Universidade Católica de São Paulo; 2006.

7. Egeli E, Ciçekci G, Silan F, Oztürk O, Harputluoglu U, Onur A et al. Etiology of deafness at the Yeditepe school for the deaf in Istanbul. Int J Pediatr Otorhinolaryngol. 2003; 67(5):467-71.

8. Ferro L, Gonçalves,I, Cieri CC. Tempo de latência entre suspeita, diagnóstico e intervenção em crianças portadoras de deficiência auditiva na cidade de Campo Grande - MS. J Bras Fonoaudiol. 2002; 3(11):108-13.

9. Heck F, Raymann BCW. Tempo decorrido entre a suspeita da surdez, a primeira ida ao médico e/ou Fonoaudiólogo, o diagnóstico e o início da reabilita- ção com crianças surdas. J Bras Fonoaudiol. 2003; 4(16):175-85.

10. Tabith A, Franco E, Barbieri J. Levantamento da etiologia da deficiência auditiva em uma escola especial para deficientes auditivos. Disturb Comun. 1989; 3:118-23.

11. Walber CA, Colomé VL, Avila SC, Balem AS, Santos, LK, Kessler TM et al. Deficiência auditiva: fatores etiológicos. Disturb Comun. 1995; 7:117-23.

12. Vartiainen E, Kemppinen $P$, Karjalainen S. Prevalence and etiology of bilateral sensorineural hearing impairment in a Finnish childhood population. Int J Pediatr Otorhinolaryngol. 1997; 41(2):175-85.

13. Zakzouk SM, Al-Anazy F. Sensorineural hearing impaired children with unknown causes: a comprehensive etiological study. Int J Pediatr Otorhinolaryngol. 2002; 64(1):17-21.

14. Wild NJ, Sheppard S, Smithells RW, Holzel H, Jones $\mathrm{G}$. Onset and severity of hearing loss due to congenital rubella infection. Arch Dis Child. 1989; 64(9):1280-3.

15. Brookhouser PE, Auslander MC, Meskan ME. The pattern and stability of postmeningitic hearing loss in children. Laryngoscope. 1988; 98(9):940-8.

16. Salerno R, Stablun G, Ceci MJ, Silva M AC. Deficiência auditiva na criança. Rev Bras Otorrinolaringol. 1985; 51:23-7.

17. Uus K, Davis AC: Epidemiology of permanent childhood hearing impairment in Estonia, 19851990. Audiology. 2000; 39(4):192-7.

18. Walch C, Anderhuber W, Köle W, Berghold A. Bilateral sensorineural hearing disorders in children: etiology of deafness and evaluation of hearing tests. Int J Pediatr Otorhinolaryngol. 2000; 53(1):31-8.

19. Billings KR, Kenna MA. Causes of pediatric sensorineural hearing loss: yesterday and today. Arch Otolaryngol Head Neck Surg. 1999; 125(5):517-21.

RECEBIDO EM: 04/09/2006

ACEITO EM: 04/08/2007

Endereço para correspondência:

Rua Graúna, 169

São Paulo - SP

CEP: 04514-000

Tel: (11) 33596234 / (11) 81226128

E-mail: lilapupo@pucsp.br 